\title{
A Randomized Trial: The Blankenstein Healthcare Plan Improves Sustainability of Lifestyle Change After Inpatient Naturopathic Therapy for Chronic Back Pain
}

\author{
Karl Rüdiger Wiebelitz*, Christa Golücke and André Michael Beer \\ Department of Naturopathy at Blankenstein Hospital, Ruhr-University Bochum, Germany
}

*Corresponding author: Karl Rüdiger Wiebelitz, Department of Naturopathy at Blankenstein Hospital, Ruhr-University Bochum, Im Vogelsang 5, D-45527, Hattingen, Germany

\section{ARTICLE INFO}

Received: 豐 November 16, 2020

Published: 幽 January 07, 2021

Citation: Karl Rüdiger Wiebelitz, Christa Golücke, André Michael Beer. A Randomized Trial: The Blankenstein Healthcare Plan Improves Sustainability of Lifestyle Change After Inpatient Naturopathic Therapy for Chronic Back Pain. Biomed J Sci \& Tech Res 33(1)-2021. BJSTR. MS.ID.005337.

Keywords: Naturopathy; Naturopathic Complex Therapy; Ordnungstherapie; Ordnungstherapy; Mind Body Medicine; Sustainability; Randomization

\section{ABSTRACT}

Ordnungstherapie is the core of 5 areas of classical European naturopathy and focuses on salutogenesis and ways to deal and live with illness. The new tool Blankenstein healthcare plan (HCP) helps to integrate health-promoting activities into the daily routine. Using the Blankenstein questionnaire (BQ) this single-center randomized controlled trial examines whether HCP improves desired lifestyle changes in patients with chronic back pain. The main outcome measure is the difference between the weighted mean values of the BQ after 3 months and at the beginning. From 2011 to 2015, 600 female patients from the Department of Naturopathy were randomized whether or not to use the HCP in addition to in-patient naturopathic complex therapy. The BQ consists of visual analogue scales for the categories of "Ordnungstherapie": conscious nutrition, exercise and sports, stress and mental stress, naysaying ability, joy of being despite illness, and care of the soul. The importance of these areas is weighted and scored individually. The weighted mean values of the BQ improved significantly after 4 weeks and 3 months in both treatment groups, but significantly more in the group with a HCP $(p<0.0001)$. No undesired effects were observed.
Abbreviations: BQ: Blankenstein Questionnaire
BMI: Body Mass Index; HCP: Healthcare Plan; VAS: Visual Analogue Scales; BDI: Beck Depression Inventory; WAVVAS: Weighted Mean Value of the VAS; CARE: Consultation and Relational Empathy; ITT: Intention to Treat; FFbH-R: Hannover Functional Ability Questionnaire Score; PP: Per Protocol; SD: Standard Deviation; LOCF: Last Observation Carried Forward; 95\%-CI: 95\%-Confidence-Interval; SLC: School Leaving Certificate; T0: At Enclosure; T1: After Inclusion in the Study; T2: At Discharge; T3: At 4 Weeks; T4: At 3 Months

\section{Introduction}

Classic European naturopathy has its place in therapy for chronic pain [1-3]. "Ordnungstherapie" (Ordnungstherapy) is the German naturopathic form of mind-body medicine. The term "Ordnungstherapie" was introduced by the Swiss physician Bircher-Benner (1867-1939) [4,5]. The focus is not on pathogenesis but is instead oriented around salutogenesis, which is an approach to human health that examines the factors contributing to the promotion and maintenance of physical and mental well-being rather than disease with particular emphasis on the coping mechanisms of the individual. Mind-body medicine works with objectively describable behavioral routines that influence spiritual and physical health. These include healthy eating and exercise routines, an appropriate lifestyle rhythm, the capability to reduce and to handle stress, successful processing of life crises and appropriate family and career structures [5]. Furthermore, the therapy includes psychosomatic, psychological, practical psychosocial and health-creating aspects and works using techniques such as autogenic training as described by Schultz [6], muscle relaxation as described by Jacobson [7], breath therapy and relaxation response.

Psychotherapeutical interventions include approaches from solution- and resource-oriented psychotherapy, behavioral therapy, short-term treatment, systemic therapy and health psychology. The resources required for the patient to manage their own illness and 
lifestyle and the coping strategies applicable for these are learned. Mind-body medicine is characterized by the concept that the specialist knowledge of the experts in the relevant areas and the experience of the patient are both necessary to achieve the goals set. In many cases, patients lack the capacity to establish a routine, that is, to create a health-promoting lifestyle that they use in their day-today life. The long-term success of a treatment is dependent on this skill [8]. Studies on the effects of lifestyle modifications triggered by mind-body medicine generally document their effectiveness and sustainability [9-11]. Success in mind-body medicine has also been shown in cardiology, oncology and pain treatment [12]. In the USA around $18.9 \%$ of patients applied mind-body medicine, which is kin to "Ordnungstherapie" [13]. Spirituality is an important resource in handling illness [14].

The Department of True Naturopathy in Blankenstein (Hattingen) has been giving intensified multimodal inpatient naturopathic complex therapy with serial individually prescribed applications at a high density since 1999. The treatment team consists of physicians, nurses, physiotherapists, Ordnungstherapists (Psychologists with further special training), ecotrophologists/ nutrition therapists and pastors. Outcomes were better for women in the naturopathy department than in an orthopedic department after 3 months. In rheumatology the results correspond with those from conventional therapies with classic disease- modifying anti-rheumatic drugs. Rehabilitation measures and treatments were performed more often following conventional orthopedic treatment. A clear additional value of naturopathy in terms of the clinical and financial aspects was seen for specific patient groups with multi-morbidities [1,15-20].

"Study findings (from exercise intervention studies) call for greater research and programmes efforts to maintain health, function, and physical activity behaviour after supports provided by research studies are removed" [21]. These observations and results from investigations on sustainability [22-24] led us to the development of a standardized form so that the process of mindbody medicine is rendered transparent and can be implemented by different therapists. To achieve this, two new therapy tools were created: the "Blankenstein questionnaire" (BQ) and the "Blankenstein healthcare plan" (HCP). Chronic back pain was selected as inclusion criterion as around $17 \%$ of the German population suffer from chronic pain, most of them are suffering from back pain - $85 \%$ at least once during their lifetime [25]. As the majority of patients treated in the Clinic for Naturopathy are women, the study is limited to female participants [26,27].

\section{Methods}

\section{Investigation Design}

1) This is a single-center randomized controlled trial with equal group size at the Clinic for Naturopathy in Blankenstein (Hattingen).
2) The patients did not receive any financial compensation.

3) The study was executed from November 2011 to August 2015.

4) Inclusion and exclusion criteria are shown in Table 1.

Table 1: Inclusion and exclusion criteria.

\begin{tabular}{|c|c|}
\hline I nclusion Criteria & Exclusion Criteria \\
\hline $\begin{array}{l}\text { Female patients from the } \\
\text { Department for Naturopathy at the } \\
\text { Blankenstein Clinic }\end{array}$ & $\begin{array}{l}\text { Participation in this study at an } \\
\text { earlier point in time }\end{array}$ \\
\hline Age $\leq 75$ years & Cachexia $(\mathrm{BMI} \leq 18.0)$ \\
\hline $\begin{array}{c}\text { FFbH-R } \leq 16 \text { points } \\
\text { (corresponding to }<70 \% \text { ) }\end{array}$ & Alcohol addiction \\
\hline $\begin{array}{c}\text { At least one of the following } \\
\text { diagnoses: cervicocephalic } \\
\text { syndrome or cervicalbrachial } \\
\text { syndrome or chronic low back pain } \\
\text { with or without radicular radiation }\end{array}$ & $\begin{array}{l}\text { Chronic pain due to other illnesses } \\
\text { which was the same as or more } \\
\text { severe than the back pain, if } \\
\text { it obliged the patient to take } \\
\text { analgesics }\end{array}$ \\
\hline $\begin{array}{l}\text { Written consent following } \\
\text { information }\end{array}$ & $\begin{array}{l}\text { Clinical signs of or anamnestic } \\
\text { clues to malignant conditions or } \\
\text { metastases in the skeleton }\end{array}$ \\
\hline \multirow[t]{3}{*}{$\begin{array}{l}\text { Sufficient length of stay (expected } \\
\qquad \geq 10 \text { days) }\end{array}$} & $\begin{array}{l}\text { Indications for surgery following a } \\
\text { specialist orthopedic investigation }\end{array}$ \\
\hline & $\begin{array}{l}\text { Patients not capable of } \\
\text { understanding the objective of the } \\
\text { investigation }\end{array}$ \\
\hline & Compliance not guaranteed \\
\hline
\end{tabular}

FFbH-R: Hannover Functional Ability Questionnaire Score

BMI: Body Mass Index

\section{Objective Criteria}

Main Outcome: Sustainability of the treatment success created by the lifestyle change, measured as difference between the WAVVAS (weighted average values of the visual analogue scales in the $\mathrm{BQ}$ : sum of the products from the relevant point number and the score on the visual analogue scale for the relevant behavior area divided by 10) after 3 months (Time T4) and the initial findings upon study inclusion (Time T1). A raise of the score is expected in both groups, more pronounced in the group with HCP.

Secondary Outcome: Sustainability of the treatment success created by the lifestyle change measured as difference between the WAVVAS after 4 weeks (Time T3) and the initial findings at Time T1. Sustainability of the treatment success produced by lifestyle changes measured using 5-step semi- quantitative scales (compare Table 8) for back pain and mental condition (subjective emotional status).

\section{Questionnaires}

Epidemiology Form: The physician documents on the epidemiology form whether the patient meets all inclusion criteria, has no exclusion criteria and whether she is participating in the study. 
BQ: This questionnaire uses visual analogue scales (VAS; therefore shortened "Blankensteiner VAS"). It assesses current health-relevant behavior in 6 different lifestyle domains and the subjective importance of a change in the near future. Repetition allows evaluation of the goals achieved and measuring the sustainability of changes. An 11-stage number bar (from 0 [very bad] to 10 [excellent]) facilitates reliable telephone questioning and discussion (Figures $1 \& 1 \mathrm{a}$ ).

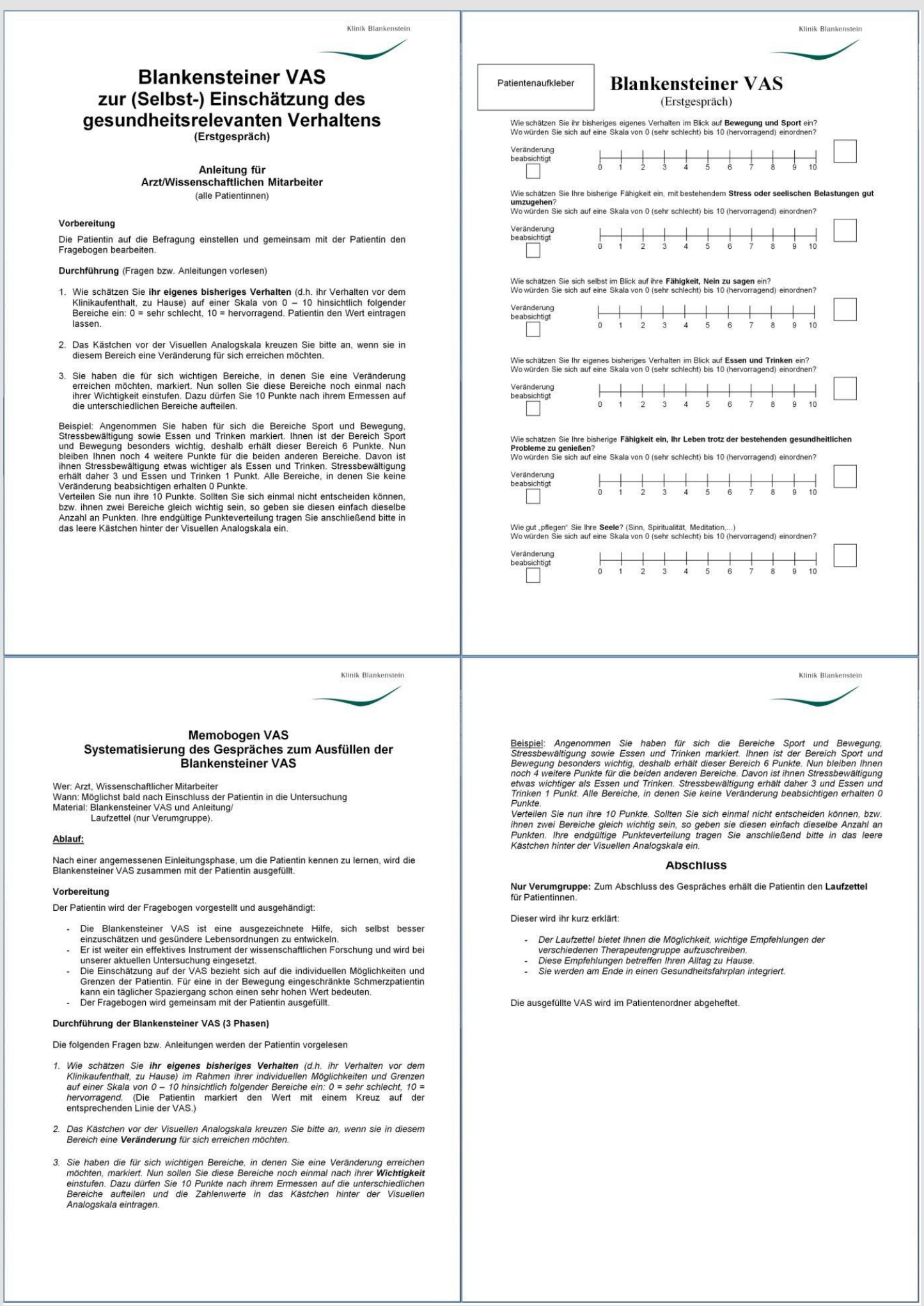

Figure 1: Blankenstein questionnaire (BQ). 




Figure 1a: Blankenstein questionnaire (BQ; English Version).

The type and extent of the implementation of the following behaviors was queried:

- Conscious handling of nutrition

- $\quad$ Movement and sport
- $\quad$ Stress and psychological burden

- $\quad$ Ability to say no

- Ability to enjoy life

- $\quad$ Looking after the soul. 
The patients worked with their therapists to establish where change was desired in each of the six areas for their individual case. A weighting of the individual significance of these areas was also carried out by allocating a total of 10 points to them. Based on this, the weighted mean value of the VAS (WAVVAS) was calculated (example: chosen areas "Ability to say no"/score 4/allocated points 7 and "Looking after the soul"/score 6/allocated points 3; WAVVAS $=(4 * 7+6 * 3) / 10=4,6)$. The minimum possible value is " 0 ", the maximum " 10 ", decimal places are allowed. The characteristics of the BQ were analyzed for the group of female in-patients $(n=600)$ with chronic back pain (not published so far): the mean value of the WAVVAS of this group is 3.84, the standard deviation 1.47, the median 3.8. The result of the WAVVAS does not correlate with age, functional impairment (Hannover Functional Ability Questionnaire $=\mathrm{FFbH}-\mathrm{R}$ ) and body mass (BMI) (correlation coefficient $<0.3$ ), only with the score of the Beck Depression Inventory (BDI) it is weakly inversely correlated (correlation coefficient -0.39; slightly lower values in case of severe depression).

Graphically, there is no indication of a non-linear correlation in any case. Nor does the duration of the complaints, intensity of the complaints, marital status, schooling, vocational training, occupation, part-time or full-time work or retirement, unemployment, past or present psychotherapeutic treatment and religiosity have any discernible influence on the WAVVAS. Only earlier participation in a health program leads to a slightly higher (0.3) mean value.

Blankenstein HCP: The Blankenstein HCP is a written plan integrating measures that promote health-relevant behavior into daily life (Figures 2 \& 2a).

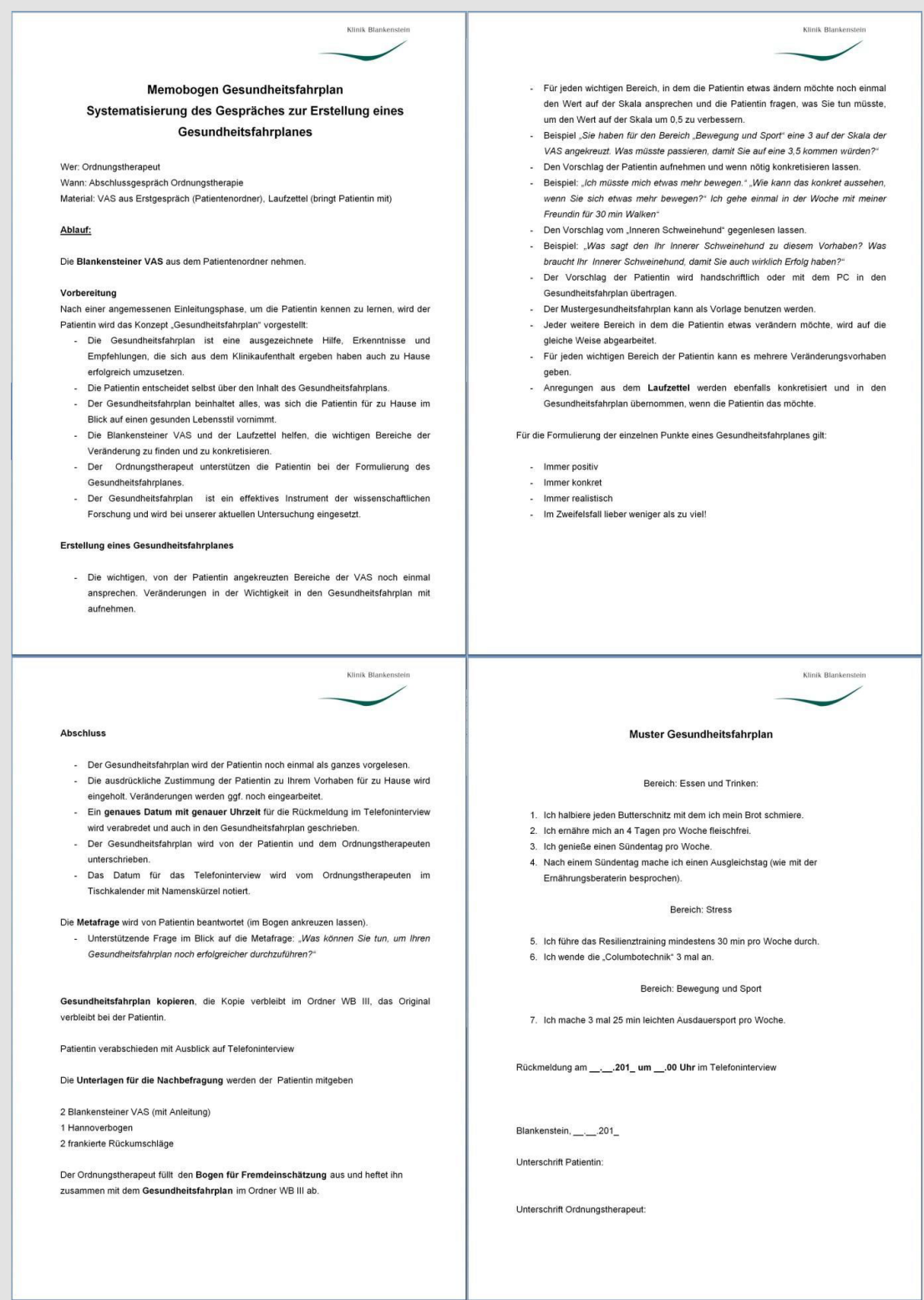

Figure 2: Blankenstein healthcare plan (HCP). 


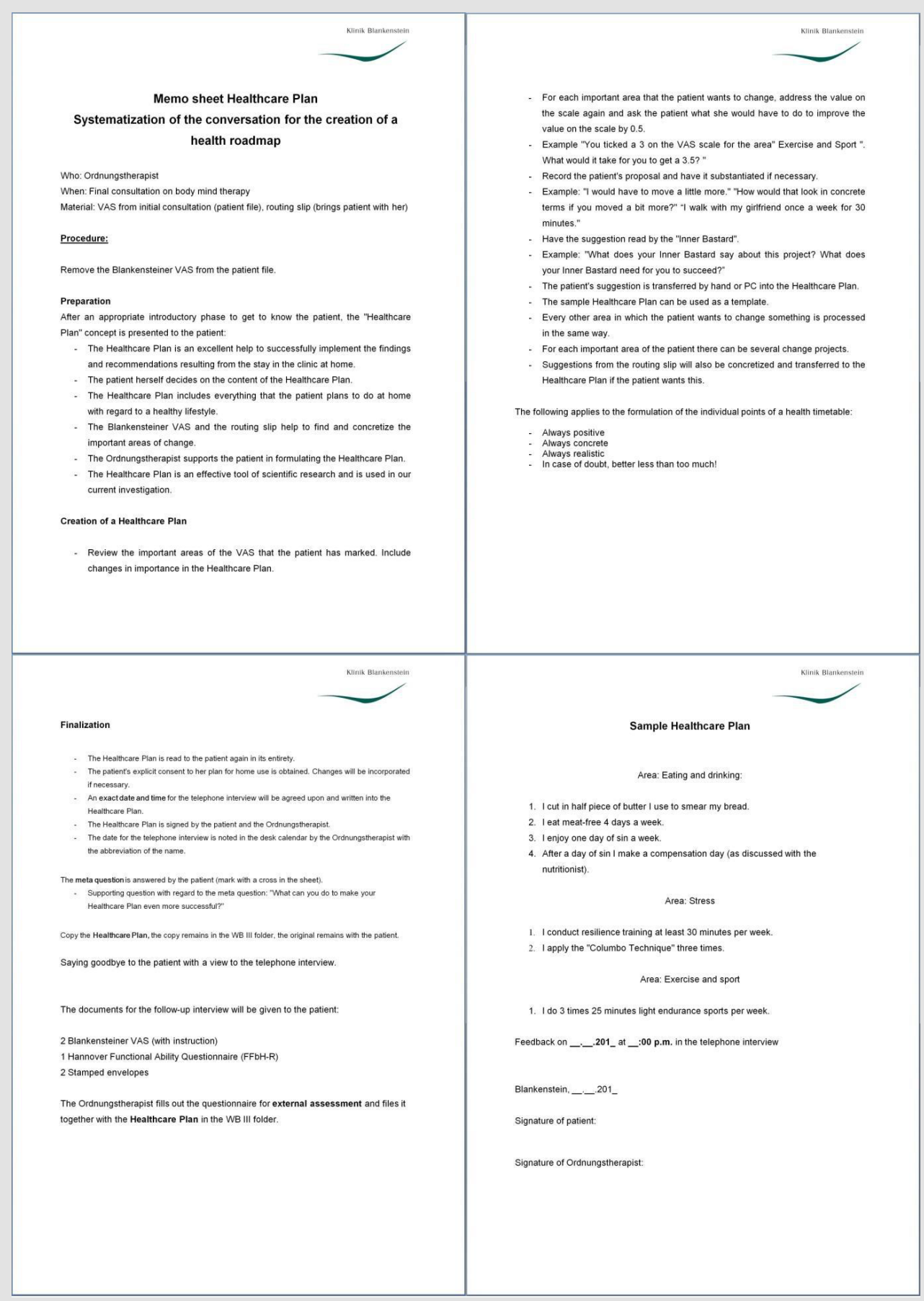

Figure 2a: Blankenstein healthcare plan (HCP; English version).

These are created individually for each patient by the patient and therapist working together based on continuous notes made during the inpatient stay. Recommendations for health-promoting measures from the therapist (physician, Ordnungstherapist, physiotherapist, nutrition therapist, care staff and pastor) are written down in these continuous notes. The plan is limited to a maximum of five measures so that it can be integrated into the daily routine. The plan is as simple and direct as possible, with the elements broken down into small steps. It focuses on creating the synergistic effect so that small successes will create an internal feeling of control and the patient will thus follow a healthy lifestyle routine. Daily team meetings provide interdisciplinary coordination. The Ordnungstherapist supports the patient in formulating the HCP. Once the HCP has been created, it is signed by both the patient and therapist, which increases the probability that it will be implemented [23]. The creation of the HCP places great value on the individuality and multidisciplinary nature of the treating team. According to studies, this is far superior to monodisciplinary and generally formulated treatment concepts [28-31]. 
a) The HCP is created by addressing the areas that the patient has indicated as important on the BQ and incorporating changes of importance into the HCP, querying for every area that a patient wants to effect change in and asking what the patient needs to do in order to improve the value on the scale by 0.5 , adopting the patient's suggestions and rendering it concrete if necessary, checking suggestions by the "devil on your shoulder",

b) Entering the patient's suggestions into the HCP, going through all other areas that the patient wants to effect change in, bearing in mind that all of the patient's important areas could have many plans for change in them,

c) Specifying the suggestions from the continuous notes and placing them in the HCP if the patient desires this, finally.

d) Reading out the complete HCP and collection explicit consent from the patient on her plans for when she returns home, incorporating changes where necessary, determining dates and times of the telephone interviews and recording them in the HCP, at last signing the HCP together with the patient.

e) The individual points of a HCP must be always positive, always concrete, always realistic and in doubt restricted to the main points (less is more).

Hannover Functional Ability Questionnaire: The FFbH-R is a standardized questionnaire to measure the physical functional impairments that occur in conjunction with the back pain (scale from $0 \%$ to $100 \%,<70 \%$ : moderate restriction of the functional capacity). Unlike the BQ, the FFbH-R does not focus on the patient's estimations but instead seeks to reflect their physical condition as objectively as possible $[32,33]$.

Beck Depression Inventory: The BDI has been used nationally [34] and internationally [35] for over 30 years. It is a psychological test that detects the severity of depressive symptoms in the clinical setting (21 questions with four possible answers each / 0-3 points; sum $\geq 29$ : severe depression). The reliability and validity are high [36] and are largely independent of age, sex and concomitant diagnoses.

\section{Standardized Telephone Interview and Written} Questionnaire: The follow-up investigations at four weeks and at three months after discharge record the implementation of the goals and measures set down in the HCP in a structured manner, based on a concrete schema (see supplementary material). The telephone interview is not carried out by the therapists themselves but by an Ordnungstherapist whom the patient does not know in order to prevent bias due to the tendency to provide a socially desirable response. The implementation of the measures in the 6 areas of the BQ is queried first, next to what percentage the goal set at discharge has been attained. Then the patient is asked how the implementation of the health-related behavior has gone overall and also whether anything else has changed in terms of the patient's well-being or general health. The BQ is filled out together.
Example: "Looking after the soul": VAS-value at the beginning: 4. Desired improvement: 2 points on the scale of 10 . Achieved improvement: $50 \%$ of the desired change. Actual VAS-value: 4 plus $50 \%$ of 2 , hence $4+0,5 * 2=5$.

Medical History Questionnaire: The medical history questionnaire is used to record the age, height, weight, initiator of the instruction, job, working hours (part/full time), level of education and diagnoses and past diagnoses.

Empathy questionnaire (CARE Questionnaire): The CARE (Consultation and Relational Empathy) questionnaire measures the physician's communication style and the empathy experienced from the physician during the consultation [37]. It was translated and validated for implementation in German-speaking countries [38] (10 questions; 5-part Likert scale). The communicative behavior of the physician influences the treatment results and the patient's satisfaction but does not significantly improve compliance $[39,40]$.

\section{Randomization}

The patients were randomly allocated to the two treatment groups by an Ordnungstherapist using a program which generates pseudo-randomized numbers whose probability is adapted to the already existing distribution (programmed with turbo pascal 4.0 using the random-function to generate pseudo-randomized results), resulting in an at least nearly equal group size. The current randomization cannot be predicted, even with electronic data processing expertise, and each randomization is documented with the patients' name, surnames and birthday in a list which the user can only read.

\section{Course of the Study}

a) The patients were selected by a physician or a research assistant using the FFbH-R and the epidemiology form and gave written informed consent.

b) 600 patients were randomized into two groups by a computer program into two equally-sized groups. One group of patients received a HCP in addition to the treatment, and the other group served as a control group.

c) During the inclusion interview the treatment process was explained, the medical history form and the BDI were filled out and the treatment goals were compiled using the BQ. The patients weighted the six areas according to their individual relevance by allocation of 10 points to the chosen areas.

d) Both groups received naturopathic complex treatment, lasting about two weeks $[2,28]$. Details of the naturopathic inpatient complex treatment were published before $[15,16,20]$.

e) In the HCP-group a continuous note form recorded the interdisciplinary recommendations of the therapists during the inpatient stay and served both as a guide and a foundation for the HCP. 
f) A CARE form was filled out upon discharge (after 2 to 3 weeks).

g) At 4 weeks and 3 months after discharge, a telephone interview recorded the implementation of the BQ and the HCP and the BQ was sent to the clinic. The FFbH-R was only filled out at 3 months after discharge.

\section{Statistics}

After a pilot phase with 20 female subjects, the clinically relevant effect was defined as a change in the WAVVAS of 0.5 (mean change of $5 \%$ with reference to the whole scale -11 point $/ 100 \mathrm{~mm}$ ) and the standard deviation was estimated. The necessary number of cases was calculated using the program $\mathrm{G}^{*}$ Power 3.0.10 for a twosided t-test for independent samples. At a desired power of $80 \%$ (type II error of 0.2) and a type I error of 0.05, at least 277 evaluable subjects are required for each group. 300 subjects were recruited for each group, based on a drop-out rate of $7 \%$ estimated by the pilot study. For the main objective parameter - difference in the WAVVAS at 3 months compared to initial findings - the differences in the WAVVAS at time T4 and at time T1 are calculated, tested for normal distribution (Shapiro-Wilk-Test) and tested between both treatment groups using the t-test for unpaired random samples. A probability of error of less than $5 \%(\mathrm{p}<0.05)$ is considered significant. The secondary outcome "difference in the WAVVAS at Times T3 and T1" is compared between the two treatment groups in the same way.

As this constitutes descriptive information, no Bonferroni correction is applied. An intention to treat analysis (ITT analysis) according to the randomization and a per protocol analysis (PP analysis) according to the actual treatment are carried out. The missing data were estimated according to the LOCF method (last observation carried forward) for the ITT analysis. Weighting points with sums different from 10 (probably because the patient deemed it inadequate to attribute the missing point to or to remove the excess point from one of the chosen categories) were standardized to 10 total weighting points. This enables weighting according to the intention of the patient. The other parameters are described by giving the mean, the standard deviation, the number of cases, student tests, $\chi^{2}$-tests and Cohens $\mathrm{d}$ for the effect size for mean differences between two groups.

\section{Institutional Review Board Approval and Trial Registration}

The study was carried out in accordance with the statements of the Declaration of Helsinki in the revised version of 1975 containing the amendments of 1983, 1989, 1996, 2000, 2004 and 2008. The version of the study as executed was approved by the Ethical Commission of the Medical Chambers of Westphalia-Lippe [Ethikkommission der Ärztekammer Westfalen-Lippe] and the Medical Faculty of the Westphalian Wilhelm University of Munster [Medizinische Fakultät der Westfälischen Wilhelms- Universität] on October 10, 2011 and November 26, 2011 (AZ 2011-222-f-S).

The study was registered in the German Clinical Trials Register (DRKS: WHO Primary Registry) (DRKS ID for the study: DRKS00003326).

\section{Results}

\section{Recruitment and Study Workflow}

The Flowchart of participant recruitment and study procedures is shown in Figure 3.

\section{Anthropometric and Epidemiological Data}

Demographic, physical and mental initial parameters for the patients are shown in Table 2. Additional anthropometric and epidemiological data are given in Tables 3-5. The level of confidence that change can be achieved is higher in the group with a HCP, both in terms of their own estimation and therapists' estimation.

Table 2: Demographic, physical and mental initial parameters.

\begin{tabular}{|c|c|c|c|c|c|c|}
\hline HCP & $56.69 \pm 10.05(\mathrm{n}=300)$ & $164.92 \pm 7.10(\mathrm{n}=285)$ & $78.56 \pm 18.03(\mathrm{n}=282)$ & $28.92 \pm 6.59(\mathrm{n}=282)$ & $11.47 \pm 3.52(\mathrm{n}=286)$ & $15.72 \pm 8.65(\mathrm{n}=288)$ \\
\hline $\mathbf{p}$ & 0.657 & 0.954 & 0.449 & 0.545 & 0.497 & 0.721 \\
\hline Total & $56.88 \pm 10.57(\mathrm{n}=600)$ & $164.93 \pm 6.76(\mathrm{n}=575)$ & $79.15 \pm 18.28(\mathrm{n}=569)$ & $29.08 \pm 6.41(\mathrm{n}=569)$ & $11.57 \pm 3.60(\mathrm{n}=576)$ & $15.59 \pm 8.22(\mathrm{n}=575)$ \\
\hline
\end{tabular}

SD: Standard deviation; BMI: Body Mass Index [kg/m2]; FFbH-R: Hannover Functional Ability Score; BDI: Beck Depression Inventory; Control: Control group without healthcare plan; HCP: Group with Blankenstein healthcare plan (HCP); p: Student's t-test for difference between both treatment groups.

Table 3: Personal status, school leaving certificate and current job.

\begin{tabular}{|c|c|c|c|c|c|}
\hline & & Total & Control & HCP & $\chi^{2}$-Test (p) \\
\hline \multirow{6}{*}{ 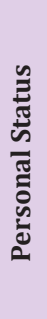 } & Single & $49(8 \%)$ & $25(9 \%)$ & $24(8 \%)$ & \multirow{6}{*}{0,86} \\
\hline & Married & $333(58 \%)$ & $163(57 \%)$ & $170(59 \%)$ & \\
\hline & Separated & $18(3 \%)$ & $9(3 \%)$ & $9(3 \%)$ & \\
\hline & Divorced & $116(20 \%)$ & $61(21 \%)$ & $55(19 \%)$ & \\
\hline & Widowed & $61(11 \%)$ & $29(10 \%)$ & $32(11 \%)$ & \\
\hline & Total & 577 & 287 & 290 & \\
\hline
\end{tabular}




\begin{tabular}{|c|c|c|c|c|c|}
\hline \multirow{10}{*}{ 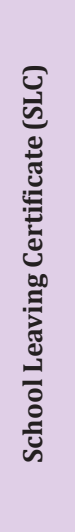 } & Still at school & $1(0,2 \%)$ & $1(\%)$ & $(\%)$ & \multirow{10}{*}{0,78} \\
\hline & No SLC & $3(0,5 \%)$ & $2(1 \%)$ & $1(\%)$ & \\
\hline & Special SLC & $7(1 \%)$ & $6(2 \%)$ & $1(\%)$ & \\
\hline & $\begin{array}{l}\text { Vocational secondary school qualifications } \\
\text { SLC }\end{array}$ & 277 (48\%) & $141(49 \%)$ & $136(47 \%)$ & \\
\hline & \multirow{2}{*}{$\begin{array}{l}\text { German General Certificate of Secondary } \\
\text { Education }\end{array}$} & & & & \\
\hline & & $182(32 \%)$ & $86(30 \%)$ & $96(33 \%)$ & \\
\hline & College or sixth form & $64(11 \%)$ & $30(10 \%)$ & $34(12 \%)$ & \\
\hline & Degree & $35(6 \%)$ & $17(6 \%)$ & $18(6 \%)$ & \\
\hline & Other & $8(1 \%)$ & $4(1 \%)$ & $4(1 \%)$ & \\
\hline & Total & 577 & 287 & 290 & \\
\hline \multirow{3}{*}{ 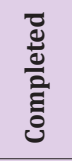 } & Yes & $507(88 \%)$ & $246(86 \%)$ & $261(90 \%)$ & \multirow{3}{*}{0,014} \\
\hline & No & $68(12 \%)$ & $40(14 \%)$ & $28(10 \%)$ & \\
\hline & Total & 575 & 286 & 289 & \\
\hline \multirow{8}{*}{ 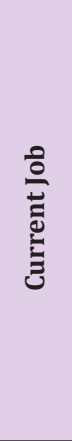 } & Without work & $88(15 \%)$ & $49(17 \%)$ & $39(13 \%)$ & \multirow{8}{*}{0,56} \\
\hline & Managerial position & $18(3 \%)$ & $9(3 \%)$ & $9(3 \%)$ & \\
\hline & Permanent contract & $221(37 \%)$ & $106(37 \%)$ & $115(38 \%)$ & \\
\hline & Temporary contract & $21(4 \%)$ & $10(3 \%)$ & $11(4 \%)$ & \\
\hline & Freelancer & $18(3 \%)$ & $8(3 \%)$ & $10(3 \%)$ & \\
\hline & Pensioner & $209(35 \%)$ & $100(34 \%)$ & $109(36 \%)$ & \\
\hline & Pension application & $18(3 \%)$ & $8(3 \%)$ & $10(3 \%)$ & \\
\hline & Total & 593 & 290 & 303 & \\
\hline \multirow{4}{*}{ 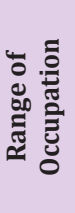 } & Full-time & $128(21 \%)$ & $63(22 \%)$ & $65(22 \%)$ & \multirow{4}{*}{0,93} \\
\hline & Part-time & $148(26 \%)$ & 73 (25\%) & 75 (26\%) & \\
\hline & None & $300(53 \%)$ & $151(53 \%)$ & $149(52 \%)$ & \\
\hline & Total & 576 & 287 & 289 & \\
\hline
\end{tabular}

SLC: school leaving certificate; HCP: Blankenstein healthcare plan

Table 4: Additional anthropometric and epidemiological data.,

\begin{tabular}{|c|c|c|c|c|}
\hline \multirow{2}{*}{ Time T1 } & & Total & Control & HCP \\
\hline \multirow{2}{*}{ (p) }
\end{tabular}




\begin{tabular}{|c|c|c|c|c|c|}
\hline \multirow{4}{*}{ 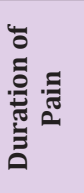 } & $<3$ months & $14(2 \%)$ & $8(3 \%)$ & $6(2 \%)$ & \multirow{4}{*}{0,63} \\
\hline & $>3<6$ months & 35 (6\%) & $18(6 \%)$ & $17(6 \%)$ & \\
\hline & $>6$ months & $527(92 \%)$ & $260(91 \%)$ & 267 (92\%) & \\
\hline & Sum & 576 & 286 & 290 & \\
\hline \multirow{4}{*}{ 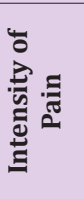 } & Low & $10(2 \%)$ & $6(2 \%)$ & $4(1 \%)$ & \multirow{4}{*}{0,53} \\
\hline & Middle & $228(39 \%)$ & $114(40 \%)$ & $114(39 \%)$ & \\
\hline & Very intense & 337 (59\%) & $165(58 \%)$ & $172(59 \%)$ & \\
\hline & SUM & 575 & 285 & 290 & \\
\hline \multirow{6}{*}{  } & Own wish & $124(22 \%)$ & $49(17 \%)$ & 75 (27\%) & \multirow{6}{*}{0,014} \\
\hline & $\begin{array}{c}\text { Recommendation of } \\
\text { physician }\end{array}$ & $258(46 \%)$ & $132(46 \%)$ & $126(45 \%)$ & \\
\hline & Both & $226(40 \%)$ & $112(39 \%)$ & $114(40 \%)$ & \\
\hline & $\begin{array}{l}\text { Recommendation of } \\
\text { a third party }\end{array}$ & $84(15 \%)$ & 36 (13\%) & 48 (17\%) & \\
\hline & Press & $34(6 \%)$ & $16(6 \%)$ & $18(6 \%)$ & \\
\hline & Sum & 566 & 284 & 282 & \\
\hline
\end{tabular}

HCP: Blankenstein healthcare plan

Table 5: Current self-assessment, desired changes across the six areas and weighting of the desired changes SD: Standard deviation;

\begin{tabular}{|c|c|c|c|c|c|c|c|c|}
\hline & & \multicolumn{2}{|c|}{$\begin{array}{c}\text { Movement and Sport } \\
\text { Mean } \pm \text { SD }\end{array}$} & $\begin{array}{l}\text { Stress and Psy- } \\
\text { chological Bur- } \\
\text { den Mean } \pm \text { SD }\end{array}$ & $\begin{array}{l}\text { Ability to say No } \\
\text { Mean } \pm \text { SD }\end{array}$ & $\begin{array}{l}\text { Conscious Han- } \\
\text { dling of Nutri- } \\
\text { tion Mean } \pm \text { SD }\end{array}$ & $\begin{array}{l}\text { Ability to Enjoy } \\
\text { Life } \\
\text { Mean } \pm \text { SD }\end{array}$ & $\begin{array}{l}\text { Looking After the } \\
\text { Soul Mean } \pm \text { SD }\end{array}$ \\
\hline \multirow{4}{*}{ 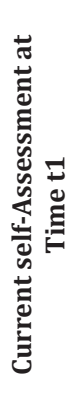 } & Control & \multicolumn{2}{|c|}{$5.227 \pm 2.509(\mathrm{n}=287)$} & $\begin{array}{l}4.548 \pm 2.127 \\
(\mathrm{n}=287)\end{array}$ & $\begin{array}{c}4.379 \pm 2.474 \\
(\mathrm{n}=287)\end{array}$ & $\begin{array}{l}5.738 \pm 2.354 \\
(\mathrm{n}=287)\end{array}$ & $\begin{array}{l}5.246 \pm 2.235 \\
(\mathrm{n}=287)\end{array}$ & $\begin{array}{c}4.888 \pm 2.525 \\
(n=286)\end{array}$ \\
\hline & HCP & \multicolumn{2}{|c|}{$5.020 \pm 2.374(\mathrm{n}=289)$} & $\begin{array}{c}4.671 \pm 2.373 \\
(\mathrm{n}=289)\end{array}$ & $\begin{array}{l}4.476 \pm 2.377 \\
(\mathrm{n}=289)\end{array}$ & $\begin{array}{l}5.596 \pm 2.238 \\
(\mathrm{n}=289)\end{array}$ & $\begin{array}{l}5.456 \pm 2.348 \\
\quad(n=289)\end{array}$ & $\begin{array}{l}4.935 \pm 2.524 \\
\quad(n=289)\end{array}$ \\
\hline & $\begin{array}{c}p \\
\text { (t-test) }\end{array}$ & \multicolumn{2}{|c|}{0.310} & 0.513 & 0.629 & 0.458 & 0.271 & 0.825 \\
\hline & Total & \multicolumn{2}{|c|}{$5.123 \pm 2.443(n=576)$} & $\begin{array}{c}4.609 \pm 2.253 \\
(\mathrm{n}=576)\end{array}$ & $\begin{array}{l}4.428 \pm 2.424 \\
\quad(n=576)\end{array}$ & $\begin{array}{c}5.667 \pm 2.295 \\
(\mathrm{n}=576)\end{array}$ & $\begin{array}{l}5.352 \pm 2.293 \\
\quad(n=576)\end{array}$ & $\begin{array}{l}4.912 \pm 2.522 \\
\quad(n=576)\end{array}$ \\
\hline \multirow{10}{*}{ 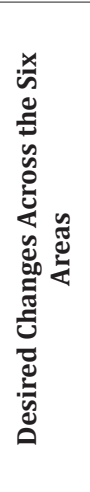 } & \multirow{3}{*}{ Control } & Yes & 160 & 202 & 166 & 156 & 151 & 146 \\
\hline & & No & 125 & 84 & 119 & 129 & 134 & 138 \\
\hline & & Total & 285 & 286 & 285 & 285 & 285 & 284 \\
\hline & \multirow{3}{*}{ HCP } & Yes & 174 & 188 & 175 & 173 & 141 & 157 \\
\hline & & No & 113 & 99 & 112 & 114 & 146 & 130 \\
\hline & & Total & 287 & 287 & 287 & 287 & 287 & 287 \\
\hline & $\chi^{2}$-Test & $\mathrm{p}$ & 0.121 & 0.069 & 0.343 & 0.056 & 0.193 & 0.261 \\
\hline & \multirow{3}{*}{ Total } & Yes & 334 & 390 & 341 & 329 & 292 & 303 \\
\hline & & No & 238 & 183 & 231 & 243 & 280 & 268 \\
\hline & & Total & 572 & 573 & 572 & 572 & 572 & 571 \\
\hline \multirow{6}{*}{ 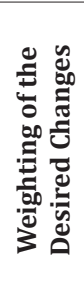 } & \multirow{2}{*}{ Control } & \multicolumn{2}{|c|}{$1.658 \pm 2.151$} & $2.260 \pm 2.121$ & $1.677 \pm 1.944$ & $1.692 \pm 2.069$ & $1.365 \pm 1.645$ & $1.378 \pm 1.863$ \\
\hline & & \multicolumn{2}{|c|}{$(n=287)$} & $(n=286)$ & $(n=286)$ & $(n=286)$ & $(n=287)$ & $(n=285)$ \\
\hline & \multirow{2}{*}{ HCP } & \multicolumn{2}{|c|}{$1.598 \pm 1.881$} & $2.043 \pm 2.021$ & $1.669 \pm 1.827$ & $2.011 \pm 2.538$ & $1.259 \pm 1.605$ & $1.467 \pm 1.753$ \\
\hline & & \multicolumn{2}{|c|}{$(\mathrm{n}=288)$} & $(n=288)$ & $(n=287)$ & $(\mathrm{n}=289)$ & $(n=285)$ & $(n=288)$ \\
\hline & $T_{T}$ & \multicolumn{2}{|c|}{$1.628 \pm 2.019$} & $2.151 \pm 2.072$ & $1.673 \pm 1.884$ & $1.852 \pm 2.320$ & $1.312 \pm 1.624$ & $1.423 \pm 1.807$ \\
\hline & Total & \multicolumn{2}{|c|}{$(n=575)$} & $(n=574)$ & $(n=573)$ & $(n=575)$ & $(n=572)$ & $(n=573)$ \\
\hline
\end{tabular}

HCP: Blankenstein healthcare plan 


\section{WAVVAS After 3 Months (Main Outcome)}

In total, 26 patients from the group with the HCP and 34 patients from the control group could not be evaluated in the PP analysis due to missing data (the reasons are given in Figure 3). One patient with HCP could not be evaluated in the PP analysis due to mixed treatment. The result of the ITT analysis and of the PP analysis of the differences in the WAVVAS (at 3 months versus baseline) with comparison of the two treatment groups is shown in Table 6.



Figure 3: Flowchart of participant recruitment and study procedures (CONSORT 2010 Flow Diagram). 
Table 6: Differences between the WAVVAS (main outcome) at times T4 (at 3 months) and T1 (after inclusion in the study) in ITT analysis and PP analysis.

\begin{tabular}{|c|c|c|c|c|c|c|c|c|}
\hline \multicolumn{2}{|c|}{ WAVVAS T4-T1 } & \multirow{2}{*}{$\begin{array}{l}\text { Mean } \\
1.326\end{array}$} & \multirow{2}{*}{$\begin{array}{c}\text { SD } \\
1.716\end{array}$} & \multirow{2}{*}{$\begin{array}{c}\mathbf{9 5} \%-\mathrm{CI} \\
1.13-1.52\end{array}$} & \multirow{2}{*}{$\begin{array}{c}\mathbf{n} \\
300\end{array}$} & \multirow{2}{*}{$\begin{array}{c}\text { d (T4-T1) } \\
0.84\end{array}$} & \multirow{3}{*}{$\begin{array}{c}\mathbf{p} \\
<0.0001\end{array}$} & \multirow[t]{2}{*}{ d $( \pm \mathrm{HCP})$} \\
\hline & Control & & & & & & & \\
\hline \multirow[t]{2}{*}{ ITT Analysis } & HCP & 1.924 & 1.686 & $1.73-2.11$ & 300 & 1.18 & & 0.35 \\
\hline & Control & 1.504 & 1.763 & $1.29-1.72$ & 266 & 0.94 & & \\
\hline PP Analysis & HCP & 2.048 & 1.644 & $1.85-2.24$ & 274 & 1.27 & $<0.0001$ & 0.32 \\
\hline
\end{tabular}

WAVVAS: weighted mean value of the visual analogue scales; SD: Standard deviation; 95\%-CI: 95\%-Confidence-Interval; ITT: Intention to treat; PP: Per Protocol; Control: Control group without healthcare plan; HCP: Group with Blankenstein healthcare plan (HCP); p: Student's t-test for difference between the two treatment groups; d: Cohens d $(0.2<\mathrm{d}<0.5$ : small effect; $0.5<\mathrm{d}<$ 0.8:medium effect; $\mathrm{d}>0.8$ : strong effect).

A positive difference between the WAVVAS at 3 months and at baseline corresponds to an improvement $(\mathrm{p}<0,001$ for both groups; ITT und PP analysis; paired t-test). The effect strength of the improvement of WAVVAS by the naturopathic complex therapy is strong in both groups. There is a small additional improvement effect in the group with HCP, which is significant (ITT: $p=0.000019$; PP: $p=0.000225$ ). The increase in the values of the WAVVAS at 3 months is greater by $45 \%$ respectively $36 \%$ with a HCP, compared to the group without HCP.

\section{WAVVAS After 4 Weeks (Secondary Outcome)}

The result of the ITT analysis and PP analysis of the differences in the WAVVAS (at 4 weeks versus baseline) with comparison of the two treatment groups is shown in Table 7.

Table 7: Differences between the WAVVAS (secondary outcome) at times T3 (at 4 weeks) and T1 (after inclusion in the study) in ITT analysis and PP analysis.

\begin{tabular}{|c|c|c|c|c|c|c|c|c|}
\hline \multicolumn{2}{|c|}{ WAVVAS T3-T1 } & Mean & SD & 95\%-CI & $\mathbf{n}$ & d (T4-T1) & $\mathbf{p}$ & $d( \pm H C P)$ \\
\hline \multirow[b]{2}{*}{ ITT Analysis } & Control & 1.179 & 1.395 & $1.02-1.34$ & 300 & 0.78 & \multirow[b]{2}{*}{0.0050} & \multirow[b]{2}{*}{0.23} \\
\hline & HCP & 1.512 & 1.499 & $1.34-1.68$ & 300 & 0.97 & & \\
\hline \multirow[b]{2}{*}{ PP Analysis } & Control & 1.349 & 1.417 & $1.17-1.51$ & 267 & 0.88 & \multirow[b]{2}{*}{0.015} & \multirow[b]{2}{*}{0.21} \\
\hline & HCP & 1.655 & 1.491 & $1.47-1.82$ & 269 & 1.06 & & \\
\hline
\end{tabular}

WAVVAS: weighted mean value of the visual analogue scales; SD: Standard deviation; 95\%-CI: 95\%-Confidence-Interval; ITT: Intention to treat; PP: Per Protocol; Control: Control group without healthcare plan; HCP: Group with Blankenstein healthcare plan (HCP); p: Student's t-test for difference between the two treatment groups; $d$ : Cohens $\mathrm{d}(0.2<\mathrm{d}<0.5$ : small effect; $0.5<\mathrm{d}<0.8$ :medium effect; d > 0.8: strong effect).

The secondary outcome increase of WAVVAS at 4 weeks is also significant higher with HCP (ITT: $p=0.0050$; PP: $p=0.015$; increase $\sim 28 \%$ respectively $\sim 23 \%$ ).

\section{Other Secondary Outcomes}

The further treatment and course results are presented for the PP analysis. The calculations were also carried out for the ITT groups, without producing significantly differing results. Change in back pain, mental well-being and FFbH-R is shown in Table 8. Change in back pain and change in mental well-being was not different between control und HCP-group at any point of time (p-values between 0.09 and 0.93; t-test) (Table 9). The FFbH-R improves significantly, with no significant difference between the treatment groups. The evaluation of the CARE form shows that the Ordnungstherapist in the group with the HCP was rated as significantly better (mean score control group 36.6, HCP-group 37.6; $\mathrm{p}=0.02$; t-test). A comparable difference cannot be detected in the evaluation of the physician (mean score control group 36.1, HCP- group 36.5; $\mathrm{p}=0.36$ ). Patients with a HCP found additional pastoral care beneficial (VAS, control group 6.7, HCP-group 8.3; $\mathrm{p}=0.007$; t-test) and the discharge letter (control group yes:165/ no:94, HCP-group yes:189/no:77; $\mathrm{p}=0.009 ; \chi^{2}$-Test) more helpful (significant at 3 months). No undesired effects were observed. 
Table 8: Change in back pain und mental well-being.

\begin{tabular}{|c|c|c|c|c|c|c|c|c|c|c|c|c|c|c|c|c|}
\hline & & \multicolumn{7}{|c|}{ Change in Back Pain } & \multicolumn{8}{|c|}{ Change in Mental Well-being } \\
\hline & &  &  &  & 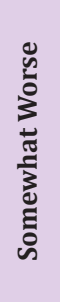 & 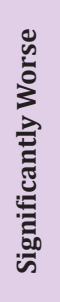 & ฮี & 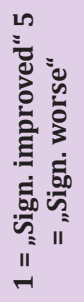 & 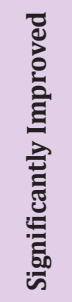 & 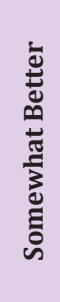 & 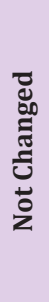 & 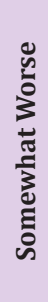 & 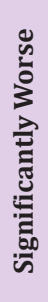 & 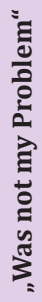 & 䒿 & 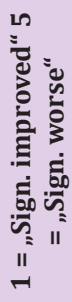 \\
\hline \multirow{3}{*}{$\begin{array}{l}\text { At Discharge } \\
\text { (T2) }\end{array}$} & Control & 80 & 175 & 9 & 0 & 0 & 264 & 1,73 & 89 & 161 & 14 & 0 & 0 & 0 & 264 & 1.72 \\
\hline & HCP & 97 & 162 & 12 & 1 & 0 & 272 & 1,69 & 101 & 161 & 6 & 0 & 1 & 3 & 272 & 1.66 \\
\hline & Total & 177 & 337 & 21 & 1 & 0 & 536 & 1,71 & 190 & 322 & 20 & 0 & 1 & 3 & 536 & 1.69 \\
\hline \multirow{2}{*}{ After } & Control & 67 & 95 & 58 & 19 & 15 & 254 & 2,29 & 71 & 103 & 58 & 17 & 7 & 0 & 256 & 2.16 \\
\hline & HCP & 60 & 102 & 67 & 12 & 18 & 259 & 2,34 & 92 & 107 & 41 & 16 & 3 & 0 & 259 & 1.96 \\
\hline 4 Weeks (T3) & Total & 127 & 197 & 125 & 31 & 33 & 513 & 2,32 & 163 & 210 & 99 & 33 & 10 & 0 & 515 & 2.06 \\
\hline \multirow{2}{*}{ After } & Control & 71 & 93 & 49 & 22 & 28 & 263 & 2,4 & 79 & 90 & 50 & 29 & 16 & 0 & 264 & 2.29 \\
\hline & HCP & 68 & 87 & 66 & 31 & 17 & 269 & 2,41 & 94 & 95 & 45 & 21 & 13 & 0 & 268 & 2.12 \\
\hline 3 Months (T4) & Total & 139 & 180 & 115 & 53 & 45 & 532 & 2,4 & 173 & 185 & 95 & 50 & 29 & 0 & 532 & 2.20 \\
\hline
\end{tabular}

Last row of each item:

Mean: 5-part Likert-scale: 1 = " Significantly improved“; 5 = "Significantly worse"

Note on the averages: 3 in the semiquantitative scale corresponds to "no change",

Values $<3$ correspond to an improvement over the previous query.

Table 9: Change in the FFbH-R.

\begin{tabular}{|c|c|c|c|c|c|}
\hline FFbH-R & Total Mean \pm SD & $\begin{array}{c}\text { Control Mean } \pm \text { SD } \\
\mathbf{9 5 \% - C I}\end{array}$ & $\begin{array}{c}\text { HCP } \\
\text { Mean } \pm \text { SD 95\%-CI }\end{array}$ & $\begin{array}{c}\text { p } \\
\text { (t-test: Con- } \\
\text { trol-HCP) }\end{array}$ & $\begin{array}{c}\text { d (Cohens d: } \\
\text { Control-HCP) }\end{array}$ \\
\hline At Enclosure T0 & $48.13 \pm 14.70$ & $48.70 \pm 14.87$ & $47.56 \pm 14.53$ & \\
\hline $\begin{array}{c}\text { After } \\
\text { 3 Months (T4) }\end{array}$ & $46.88-49.37(\mathrm{n}=536)$ & $46.91-50.49(\mathrm{n}=266)$ & $45.23-48.85(\mathrm{n}=270)$ & 0.370 & -0.077 \\
\hline $\begin{array}{c}\mathbf{p} \\
\text { (t-test: T0-T4) }\end{array}$ & $56.94 \pm 20.84$ & $56.80 \pm 21.25$ & $57.08 \pm 20.47$ & 0.877 & \\
\hline $\begin{array}{c}\mathbf{d} \\
\text { (Cohens d: T0-T4) }\end{array}$ & $<0.0001$ & $54.25-59.35(\mathrm{n}=266)$ & $54.41-59.33(\mathrm{n}=272)$ & & \\
\hline
\end{tabular}

FFbH-R: Hannover Functional Ability Score; d: effect size $(0.2<\mathrm{d}<0.5$ : small effect; $0.5<\mathrm{d}<0.8$ :medium effect; $\mathrm{d}>0.8$ : strong effect); SD: Standard deviation; 95\%-CI: 95\%-Confidence-Interval.

\section{Discussion}

Randomization led to comparable treatment groups without relevant differences in the starting parameters for both groups. Limitations of the study and its statements result from the restriction to the female gender, so that a possible transferability to men remains unclear. It also remains unclear whether the HPC can also be used effectively in the outpatient area. The level of confidence that change can be achieved is higher in the group with a HCP, both in terms of the patients and the therapists' estimation.
It could be that bias has an effect here as the patients and the care providers knew about the group assignment at this point and potentially hoped that they would benefit from the HCP being tested. The better result of the Ordnungstherapist in the CARE form for the group with the HCP may be due to the increased intensity of care that the group with the HCP experienced. As we have seen from both previous scientific monitoring sessions, naturopathic complex therapy improves the functional impairments caused by back pain in both treatment groups, and therefore for the population as a 
whole. This was measured with the FFbH-R over the course of the 3 month observation period and is significant.

No difference between the treatment groups could be evidenced here. Both treatment groups and therefore the entire study population had significant treatment success from the naturopathic complex therapy, as evidenced by the parameters measured by the BQ at all times. The effect size is strong. Whether the new use of the measuring instrument BQ itself is at least partly responsible for this cannot be decided with the current study design. The HCP leads to a significant and quantitatively meaningful increase in treatment success in both the PP analysis and the ITT analysis. Because the follow-up period was limited to 3 months, the long-term generalization of the results remains a task for future studies. It also remains to be seen whether the improvement in back pain with HCP lasts longer in the long term, so that the results of the Hannoveraner Bogen and measurements of the quality of life not directly and validated examined in this study also lead to demonstrable significant differences after a few years.

\section{Conclusion}

The HCP, used in conjunction with the $\mathrm{BQ}$ is a valuable instrument in securing long-term success of mind- body medicine ("Ordnungstherapie"). Intended changes in Lifestyle are more intense and persisting. The HCP in conjunction with the BQ should be integrated into the routine care of naturopathic patients with chronic back pain, despite the significant increase in personal workload that this brings for the care provider. The question of whether these results could also be applied to naturopathic patients with other chronic diseases and to conventional medical departments, potentially in a form adapted specifically for the department, particularly if the departments are offering a form of complex therapy (such as pain therapy), should be explored in future studies.

\section{Acknowledgement}

All members of the therapeutic team of the naturopathic department, particularly the psychologists and the physicians, contributed to the success of this study.

\section{Informed Consent and Patient Details}

We confirm all patient/personal identifiers have been removed or disguised so the patient/person(s) described are not identifiable and cannot be identified through the details of the story.

\section{Conflict of Interest}

All authors declare that no conflicts of interest exist.

\section{Funding Statement}

This research did not receive any specific grant from funding agencies in the public, commercial, or not- for-profit sectors.

\section{References}

1. Beer AM, Ostermann T, Matthiessen PF (2001) Changes in quality of life during acute inpatient naturopathic treatment--results of the Blankenstein model. Gesundheitswesen 63(4): 242-247.

2. Beer AM, Stockheim M, Fey S (2006) Chronic back pain. Naturopathy and Conventional Medicine in Direct Comparison. Praxis Magazin 12: 22-29.

3. Ostermann T, Langhorst J, Beer AM (2013) The Effects of Integrative InPatient Treatment on Patients' Quality of Life: A Meta-Analysis. Review Article. Evid Based Complement Alternat Med pp. 416510.

4. Neuendorff F, Beer AM (2006) Attempt to Determine the Position: Ordnungstherapy in an Interdisciplinary Comparison. Erfahrungsheilkunde 55: 603-606.

5. Melzer J, Melchart D, Saller R (2004) Development of Ordnungstherapy by Bircher-Benner in Naturopathy in the 20th Century. Forsch Komplementarmed Klass Naturheilkd 11(5): 293-303.

6. Schultz JH (1960) Psychotherapy and autogenic training. Arztl Forsch 14: I/325-I/326.

7. Jacobson EA (1938) Progressive relaxation; a physiological and clinical investigation of muscle states and their significance in psychology and medical practice. University of Chicago Press, Chicago, USA.

8. Dobos G, Paul A (2011) Mind-Body Medicine. (2 $2^{\text {nd }}$ Edn.). Elsevier, Urban \& Fischer Verlag, München, Deutschland.

9. Langhorst J, Elsenbruch S, Müller T, Popkirowa K, Lüdtke R, et al. (2004) Intensified lifestyle modification in patients with ulcerative colitis: effects on quality of life, psychoneuroimmunological variables and inflammatory markers in stool. Z Gastroenterol 42: 118.

10. Michalsen A, Dobos G (2005) Heart Rate Reduction through Lifestyle Change. Eur Heart J 26(17): 1806-1807.

11. Michalsen A, Grossman P, Lehmann N, Knoblauch NTM, Paul A, et al. (2005) Psychological and quality-of-life outcomes from a comprehensive stress reduction and lifestyle program in patients with coronary artery disease: results of a randomized trial. Psychother Psychosom 74: 344352 .

12. Astin JA, Shapiro SL, Eisenberg DM, Forys KL (2003) Mind-Body Medicine: State of the Science, Implications for Practice. J Am Board Fam Med 16(2): 131-147.

13. Wolsko PM, Eisenberg DM, Davis RB, Phillips RS (2004) Use of Mind Body Medical Therapies Results of a National Survey. J Gen Intern Med 19(1): 43-50.

14. Büssing A, Ostermann T, Matthiessen PF (2007) Adaptive Coping and Spirituality as a Resource in Cancer Patients. Breast Care 2: 195-202.

15. Beer AM, Ostermann T, Matthiessen PF (2001) Evaluation of Stationary Naturopathy. The Blankenstein model. Part I: Patient Clientele and Therapeutic Concepts. Forsch Komplementärmed 8(1): 6-13.

16. Ostermann T, Beer AM, Matthiessen PF (2002) Evaluation of Inpatient Naturopathy - The Blankenstein Model: Part II: Effect Sizes and Health Status of Patients over Time. Forsch Komplementärmed 9(5): 269-276.

17. Beer AM (1999) Model Trial on Natural Remedies in the Inpatient Area, in: S. Moebus, (Edi.), Scientific Support of Model Projects on Natural Remedies. Essen: Forum Medizin, p. 3-4.

18. Ostermann T, Matthiessen PF (2005) Naturopathy in the Stationary Acute Care. Evaluation of the Blankenstein Model. VAS, Frankfurt, Deutschland.

19. Wiebelitz KR, Teske W, Henke T, Knobloch R, Winnemöller C, et al. (2010) Comparison of treatment expenses of naturopathic and orthopedic inpatient treatment. MMW Fortschr Med 151 Suppl 4: 159-168.

20. Wiebelitz KR, Teske W, Henke T, Brach J, Beer AM (2011) Naturopathic and orthopaedic in-patient treatment of chronic back pain--a comparison study. MMW Fortschr Med 153 Suppl 2: 47-55. 
21. Lai B, Kim Y, Wilroy J, Bickel CS, Rimmer JH, et al. (2019) Sustainability of exercise intervention outcomes among people with disabilities: a secondary review. Disabil Rehabil 41(13): 1584-1595.

22. Bonsack S, Spirig R, Baldegger E, Wettstein S (2006) Results of the Qualitative Evaluation of Allfit - a Training and Exercise Program for the Chronically ill Elderly: The Mode of Action from the Perspective of Participants in Diabetes or Rheumatic Courses. Pflege 19(4): 244-250.

23. Deck R, Häppe A, Arlt AC (2009) Improvement of Rehabilitation Aftercare through long term follow along of the patients - Results of a pilot study. Rehabilitation 48(1): 39-46.

24. Dibbelt S, Greitemann B, Büschel C (2005) Long-term Efficiency of Orthopedic Rehabilitation in Chronic Back Pain--The Integrative Orthopedic Psychosomatic Concept (IopKo). Rehabilitation 45(6): 324335.

25. Bundesärztekammer (BÄK) (2017) Kassenärztliche Bundesvereinigung (KBV), Arbeitsgemeinschaft der Wissenschaftlichen Medizinischen Fachgesellschaften (AWMF). Nationale VersorgungsLeitlinie Nichtspezifischer-Kreuzschmerz - Kurzfassung [National Care Guideline NonSpecific-Low Back Pain - Short Version], (2 ${ }^{\text {nd }}$ Edn.)., Version 1.

26. Schwartz JB (2000) Gender differences in response to drugs: pain medications J Gend Specif Med 2(5): 28-30.

27. Teuber N, Thiele A, Eberhardt B (2006) Gender Role and Pain Experience. Schmerz 20(4): 307-313.

28. Beer AM, Uehleke B, Wiebelitz KR (2013) The history of inpatient care in German departments focusing on natural healing. Review Article. Evid Based Complement Alternat Med pp. 521879.

29. Cecchi F, Gasquini G, Papaerini A, Boni R, Castagnoli C, et al. (2014) Predictors of response to exercise therapy for chronic low back pain result of a prospective study with one year follow-up. Eur J Phys Rehabil Med 50(2): 143-151.

30. Silvemark A, Källmén H, Molander C (2014) Improved life satisfaction and pain reduction: Follow- up of a 5-week multidisciplinary long-term pain rehabilitation programme. Ups J Med Sci 119(3): 278-286.

\section{ISSN: 2574-1241}

DOI: 10.26717/BJSTR.2021.33.005337

Karl Rüdiger Wiebelitz. Biomed J Sci \& Tech Res

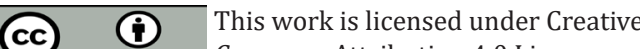
Commons Attribution 4.0 License

Submission Link: https://biomedres.us/submit-manuscript.php
31. Waterschoot FPC, Dijkstra P, Hollak N, De Vries HJ, Geertzen JHB, et al. (2014) Dose or content? Effectiveness of pain rehabilitation programs for patients with chronic low back pain: A systematic review. Pain 155(1): 179-189.

32. Kohlmann T, Raspe H (1996) Hannover Functional Questionnaire in ambulatory diagnosis of functional disability caused by backache. Rehabilitation 35(2): I-VIII.

33. Haase I, Schwarz A, Burger A, Kladny B (2001) The Function Questionnaire Hannover (FFbH) and the Subscale "Physical Functioning" from the Sf-36 in Comparison. Rehabilitation 40(1): 40-42.

34. Hautzinger M, Bailer MH, Keller F (1995) Beck-Depressions-Inventar (BDI). [Beck-Depression-Inventory (BDI)]. Bern: Huber.

35. Beck AT, Steer RA, Brown GK (1996) Beck depression inventory. TX: The Psychological Corporation, San Antonio, USA

36. Richter P, Werner J, Heerlein A, Kraus A, Sauer H (1998) On the validity of the Beck Depression Inventory. A Review. Psychopathology 31: 160168.

37. Mercer SW, Maxwell M, Heaney D, Watt GCM (2004) The consultation and relational empathy (CARE) measure: Development and preliminary validation and reliability of an empathy-based consultation process measure. Fam Pract 21(6): 699-705.

38. Neumann M, Wirtz M, Bollschweiler E, Warm M, Wolf J, et al. (2008) Psychometric evaluation of the German version of the measuring instrument "Consultation and Relational Empathy" (CARE) on the example of cancer patients. Psychother Psych Med 58(1): 5-15.

39. Di Matteo MR (2004) Variations in patients' adherence to medical recommendations: A quantitative review of 50 years of research. Med Care 42(3): 200-209.

40. Di Matteo MR, Lepper HS, Croghan TW (2000) Depression is a Risk Factor for Noncompliance with Medical Treatment. Meta-analysis of the Effects of Anxiety and Depression on Patient Adherence. Arch Intern Med 160(14): 2101-2107.

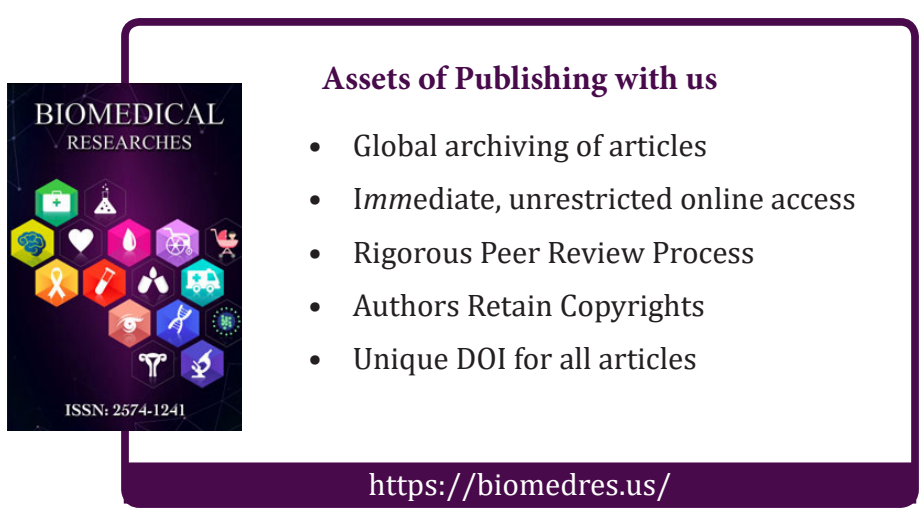

